\title{
The influence of probiotics on genital high- risk human papilloma virus clearance and quality of cervical smear: a randomized placebo-controlled trial
}

\author{
Yu-Che Ou ${ }^{1}$, Hung-Chun Fu ${ }^{2}$, Chih-Wen Tseng ${ }^{1}$, Chen-Hsuan Wu ${ }^{2}$, Ching-Chou Tsai ${ }^{2}$ and Hao Lin ${ }^{2^{*}}$ (D
}

\begin{abstract}
Background: Probiotics has been shown to be effective in reducing vaginal colonization of pathogenic organisms. The aim of this study was to investigate the influence of probiotic strains Lactobacillus rhamnosus GR-1 and Lactobacillus reuteri RC-14 on genital high-risk human papilloma virus (HR-HPV) clearance and quality of cervical smear.

Methods: This was a randomized, double-blinded, placebo-controlled trial. Women with genital HR-HPV infection were randomized into study and control groups. A probiotic or placebo preparation was administered orally (one capsule daily) until negative HR-HPV testing. A cervical smear and HR-HPV tests were performed at the beginning of the study and every 3 months thereafter until a negative result was obtained.

Results: A total of 121 women with genital HR-HPV infection were enrolled (62 in the study group and 59 in the control group). There was no significant difference in HR-HPV clearance rate between the two groups (58.1\% vs. 54.2\%). The only factor predicting HR-HPV clearance was a lower initial viral load (HR 3.214; 95\% Cl: 1.398, 7.392; $p=$ 0.006). Twenty-two women had a mildly abnormal initial cervical smear and nine had an unsatisfactory smear. At 6 months follow-up, both mildly abnormal cervical smear and unsatisfactory smear rates had decreased significantly in the study group compared to the control group ( $p=0.017$ and 0.027 ).

Conclusions: The application of probiotic strains Lactobacillus rhamnosus GR-1 and Lactobacillus reuteri RC-14 did not influence genital HR-HPV clearance, but may have decreased the rates of mildly abnormal and unsatisfactory cervical smears.
\end{abstract}

Trial registration: Clinicaltrials.gov NCT01599416, May, 2012. Retrospectively registered.

Keywords: Lactobacillus rhamnosus, Lactobacillus reuteri, Human papilloma virus, Cervical smear

\section{Background}

A healthy vaginal microbiome, mostly containing lactobacilli microorganisms, can aid in the prevention of bacterial vaginosis (BV), fungal infections and other possible problems by maintaining an acidic $\mathrm{pH}(<4.5)$ that is unfavorable for the growth of common pathogens.

\footnotetext{
* Correspondence: haolin423700@gmail.com; haolin@adm.cgmh.org.tw 2Department of Obstetrics and Gynecology, Kaohsiung Chang Gung Memorial Hospital and Chang Gung University College of Medicine, 123, Ta Pei Road, Niao Sung District, Kaohsiung City 83301, Taiwan, Republic of China

Full list of author information is available at the end of the article
}

The reduction or absence of vaginal lactobacilli has been shown to be a major cause of $\mathrm{BV}$, and to increase the risks of vaginitis and recurrent urinary tract infections by $2-4$ times $[1,2]$. In addition, harmful bacteria transmitted via sex or an imbalance in bacteria may cause a change in $\mathrm{pH}$ in the vagina, thereby presenting a suitable environment for virus infection and expansion [3, 4]. Furthermore, a positive association between BV and cervical high-risk human papilloma virus (HR-HPV) infection was suggested in a meta-analysis by Gillet et al. [5].

Epidemiologic studies have indicated that HR-HPV is the main etiological agent in the development of cervical

(c) The Author(s). 2019 Open Access This article is distributed under the terms of the Creative Commons Attribution 4.0 International License (http://creativecommons.org/licenses/by/4.0/), which permits unrestricted use, distribution, and reproduction in any medium, provided you give appropriate credit to the original author(s) and the source, provide a link to the Creative Commons license, and indicate if changes were made. The Creative Commons Public Domain Dedication waiver (http://creativecommons.org/publicdomain/zero/1.0/) applies to the data made available in this article, unless otherwise stated. 
cancer. However, only $10 \%$ of HR-HPV infections persist and potentially progress to cervical cancer [6]. It is unknown why HR-HPV infection is cancerous in some women but eradicated in others. Individual differences in immunological defense may be one explanation, and local cervical factors may also determine the outcome of HR-HPV infection [6, 7].

The quality and sensitivity of a cervical cytological diagnosis can be affected by the presence of vaginal infections due to the accumulation of numerous microorganisms, white blood cells, and degradation products. In order to improve the reliability of cervical cytology, treatment of vaginal infections and maintaining a healthy vaginal environment are necessary. U-relax ${ }^{\circ}(\mathrm{U}-$ relax, Tri-factor Biotech Inc., Taiwan), an oral probiotic used to restore vaginal flora, contains two patented and clinically proven probiotic strains: Lactobacillus rhamnosus GR-1 and Lactobacillus reuteri RC-14. Reid et al. had reported evidence that these two probiotic strains can be delivered to the vagina following oral intake via morphology identification and molecular typing [8]. Clinically, oral U-relax ${ }^{\oplus}$ has been shown to be safe and effective in reducing vaginal colonization of pathogenic bacteria and yeast [9-11]. However, the association between these probiotics and HR-HPV infection has not been fully investigated. Therefore in present study, we attempted to evaluate the influence of oral U-relax ${ }^{\circ}$ on genital HR-HPV clearance and quality of cervical smear diagnosis.

\section{Methods}

The study was a randomized, double-blinded, placebocontrolled clinical trial. Participants were recruited at Kaohsiung Chang Gung Memorial Hospital from January 2010 through June 2013. After Institutional Review Board approval, informed consent was obtained from all patients. The inclusion criteria were 1) females aged 30 to 65 years with HR-HPV infection, 2) cervical smear test with negative for intraepithelial lesion or malignancy results, 3) not pregnant. The exclusion criteria included 1) cervical cancer patients, 2) cervical intraepithelial neoplasia before conization, and within 6 months after conization, 3) gastrointestinal dysfunction or prior history of gastrointestinal surgery. All women received cervical smears and HR-HPV testing to confirm the HRHPV infection. A colposcopic biopsy was performed in patients with cervical cytology of ASCUS and above.

In order to achieve $75 \%$ power at a 0.05 significance level, an overall sample size of at least 180 subjects (90 in the control and 90 in the treatment group) should be enrolled. However, enrollment was slow therefore a decision of closing accrual was made 2 years after study commenced. Randomization was performed through application program provided at the website (http://www. randomization.com/) using a computerized, balanced (1:1) method. Random numbers were generated by a computer, and the randomization code was inserted into numbered, sealed, opaque envelopes. A single envelope was opened by the patient when they were included. Study coordinators, patients, gynecologists and members of the panel were masked to the intervention after assignment. The study group was treated orally with one capsule a day of U-relax (U-relax, Tri-factor Biotech Inc., Taiwan). Each capsule contains $180 \mathrm{mg}$ of a standardized, light beige fine powder (glucose anhydrate, potato starch, microcrystalline cellulose and magnesium stearate) consisting of freezedried cultures (50\% Lactobacillus rhamnosus GR-1 and $50 \%$ Lactobacillus reuteri RC-14). Quality control on the product showed that each capsule of U-relax has a minimum potency of 5.4 billion $(5.4 \mathrm{E}+9) \mathrm{CFU}$ (Colony Forming Units) and can be stored in room temperature 5$30 \mathrm{C}$ without dramatic change in CFU. The control group received the same capsule contains $180 \mathrm{mg}$ of a standardized, light beige fine powder but without any probiotic bacteria. The treatment was discontinued until negative HR-HPV testing.

Cervical swabs from the uterine cervix and endocervix were obtained every 3 months for cytology examinations and also for HR-HPV testing until a negative result was obtained. For cervical cytology, the swab was transferred to a glass microscope slide glass, fixed in $95 \%$ ethanol and tinted using Papanicolaou (hematoxylin, methyl orange, and polychrome). The preparations were then analyzed for abnormal cells by cytopathologists, and the results were classified according to the Bethesda System. Inflammation was defined as an increased number of polymorphonuclear leukocytes or neutrophils and parabasal cells with generalized eosinophilia of the cells. Unsatisfactory result was defined as more than $75 \%$ of the epithelial cells were obscured or could not be clearly visualized. For HR-HPV testing, we used Hybrid Capture 2 (HC2) test kits (Digene, Silver Spring, MD, USA) according to the manufacturer's protocol. Samples with a relative light unit (RLU) ratio higher than 1.0 were recorded as positive, and viral load was defined as RLU/ positive controls.

Age, parity, menopausal status, educational level, employment status, prior hysterectomy, intrauterine device (IUD) use, viral load, HPV clearance, and cervical smear results were compared between the two groups. Data obtained from this study were analyzed using the chisquare test, Fisher's exact test and Student's $t$-test. Hazard ratios (HRs) were estimated from multivariate logistic regression models to identify the independent factors predicting HPV clearance. The Kaplan-Meier method was used to estimate the time to clearance of HPV infection. All data were analyzed using SPSS statistical software version 22 (IBM Institute, Cary, NC, USA). Results 
with a $p$ value $<0.05$ were considered to be statistically significant.

\section{Results}

Finally a total of 141 women were assessed for eligibility. Five were excluded due to not meeting inclusion criteria or declined to participate, and the remaining 136 women with HR-HPV infection were enrolled and randomly allocated to the study (68 women) and control (68 women) groups. A two-sided log rank test achieved $55.8 \%$ power at this sample size. During followed up period, 6 and 9 women in the study and control groups, respectively, were further excluded for analysis due to lost follow-up or stopped treatment. The CONSORT flow diagram is shown in Fig. 1. The patients' demographic data are summarized in Table 1 . There were no significant differences in age, parity, menopausal status, educational level, employment status, prior total hysterectomy history, intrauterine device (IUD) use, pretreatment viral load, and HR-HPV clearance between the study and control groups.

With regards to viral clearance, HR-HPV was cleared in $33.1,43.8,47.9$, and $56.2 \%$ of the entire cohort at 3,6 , 9 , and 12 months follow-up, respectively. The clearance rate was similar between the study $(58.1 \%)$ and control (54.2\%) groups. Figure 2 shows the Kaplan-Meier curves of the two groups for the estimated time to clearance of HR-HPV infection. There was no difference in the time to clearance between the two groups (log rank test, $p=0.741)$. Age, menopausal status, educational level, employment status, prior hysterectomy, intrauterine device (IUD) use, and the use of probiotics were not associated with viral clearance. However, the women with HR-HPV clearance had significantly lower mean parity $(p=0.03)$ and lower initial viral load $(p=0.019)$. In multivariate logistic regression analysis, a lower initial HR-HPV viral load was the only independent factor predicting viral clearance after adjusting for confounding factors (HR 3.214, 95\% confidence interval: 1.398, 7.392; $p=0.006$ ) (Table 2).

Cervical cytological findings before and after therapy are presented in Table 3. Overall, 22 women initially had a mildly abnormal cytological diagnosis (11 ASCUS and 11 LSILs), and nine women had unsatisfactory results. The unsatisfactory rate was rather high in our study, mostly due to excessive inflammatory cells rather than inadequate sampling. There were no significant differences between the study and control groups before treatment in terms of inflammation, ASCUS, LSILs, and unsatisfactory results. There were no significant changes in the cytological findings between the two groups at 3 months follow-up. However, at 6 months follow-up, only 10 and 6 women had a mildly abnormal cytology and unsatisfactory results, respectively. There were a significant decrease in ASCUS/LSILs in the study group (13 to 4$)$ compared to the control group (9 to 6) $(p=0.017)$. The unsatisfactory cases also dramatically dropped from 5 to 2 in the study group while there was no change in the control group

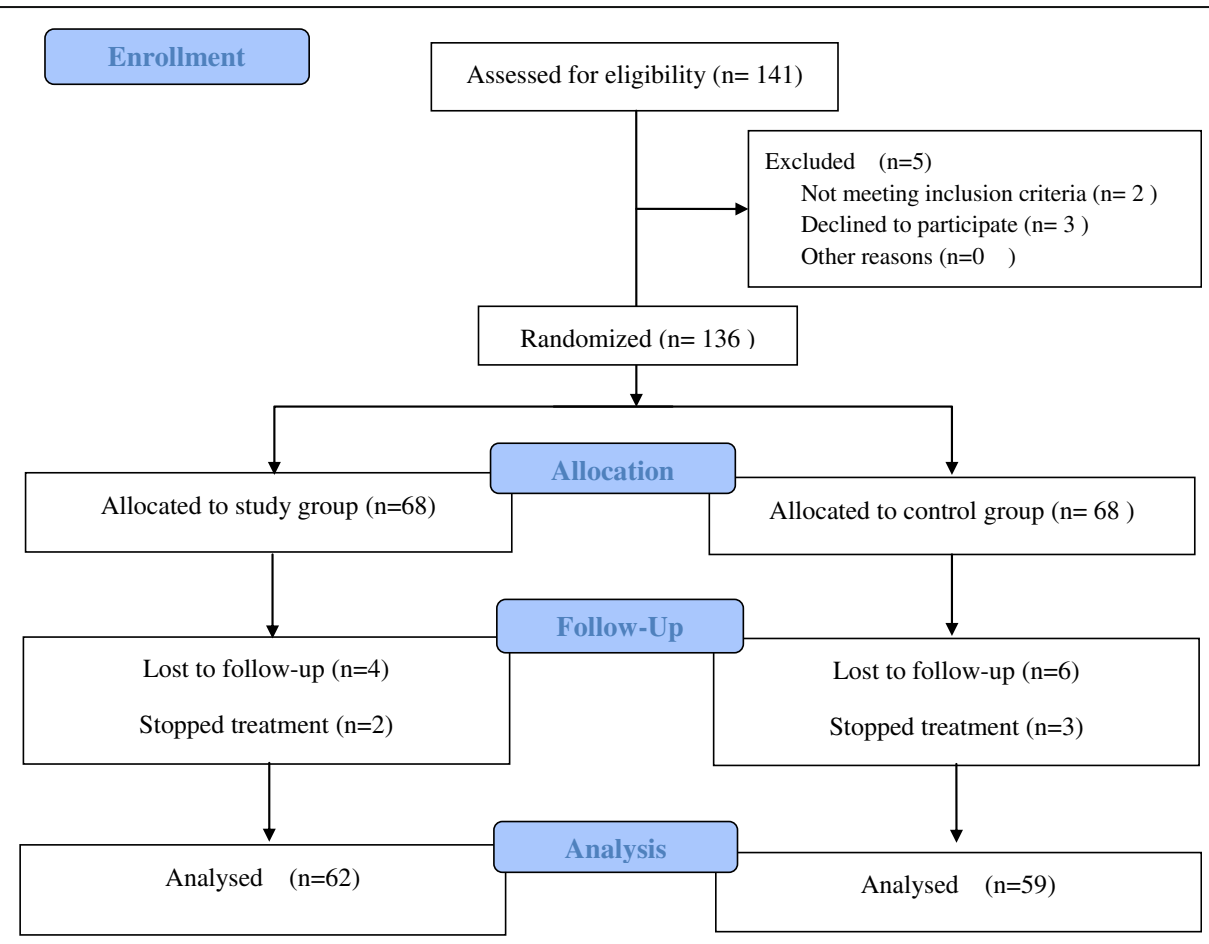

Fig. 1 Consort flow diagram 
Table 1 Demographic characteristics of the study and control groups

\begin{tabular}{llll}
\hline & Study Group N=62 & Control Group N=59 & $p$-value \\
\hline Age, years (mean \pm SD) & $45.81 \pm 10.83$ & $43.83 \pm 11.06$ & 0.897 \\
Post-menopause & $18(29.0 \%)$ & $19(32.2 \%)$ & 0.839 \\
Parity & $1.91 \pm 1.05$ & $2.12 \pm 1.23$ & 0.327 \\
IUD & $8(12.9 \%)$ & $4(6.7 \%)$ & 0.268 \\
Educational level (college or above) & $22(35.5 \%)$ & $17(28.8 \%)$ & 0.652 \\
Employed & $42(67.7 \%)$ & $36(61.0 \%)$ & 0.559 \\
History of TH & $4(6.5 \%)$ & $7(11.9 \%)$ & 0.355 \\
HR-HPV load (RLU/PC) (mean \pm SD) & $427.08 \pm 771.50$ & $416.06 \pm 719.51$ & 0.686 \\
HR-HPV clearance & $36(58.1 \%)$ & $32(54.2 \%)$ & 0.716 \\
ASCUS/LSIL & $13(21.0 \%)$ & $9(15.3 \%)$ & 0.522
\end{tabular}

ASCUS/LSIL atypical squamous cell of undetermined significance/low-grade squamous intraepithelial lesion, $H R$ - $H P V$ high-risk human papilloma virus, IUD intrauterine device, $R L U / P C$ relative light unit/positive control, SD standard deviation, $T H$ total hysterectomy

( $p=0.027)$. The cytological findings at 9 and 12 months remained similar between the two groups. No progression of cytological results was noted throughout the study period.

\section{Discussion}

The rationale of using probiotic strains in HPV clearance is via three proposed mechanisms. First, a synergistic environment is created. The anti-microorganism effects of probiotic strains are through space competition, nutrition competition, and the production of inhibitory compounds (biosurfactants, hydrogen peroxide, lactic acid, bacteriocins, and coaggregation molecules) [12]. Thus an increased number of probiotic strains in the vagina may be able to prevent and reduce HPV infections or expansion. Second, enhanced innate and adaptive immunity, which is the major defense mechanism against viral infections. Many studies have reported that immunization with the probiotics GR-1 and RC-14 can increase CD4 count in patients with acquired immune deficiency syndrome (AIDS) [13], and regulate TNF (tumor necrosis factor)alpha, IL (interleukin)-6, IL-8, IL-10 and IL-12 (p70) in the neurogenic bladders of patients with spinal cord injuries with urinary tract infections [14]. Third, through a direct antiviral effect via the secretion of specific metabolites [15]. Cadieux et al. found that probiotic strains had the ability to inactivate viruses [16]. Cha et al. also showed an antiviral activity on HPV type 16 through suppression of E6

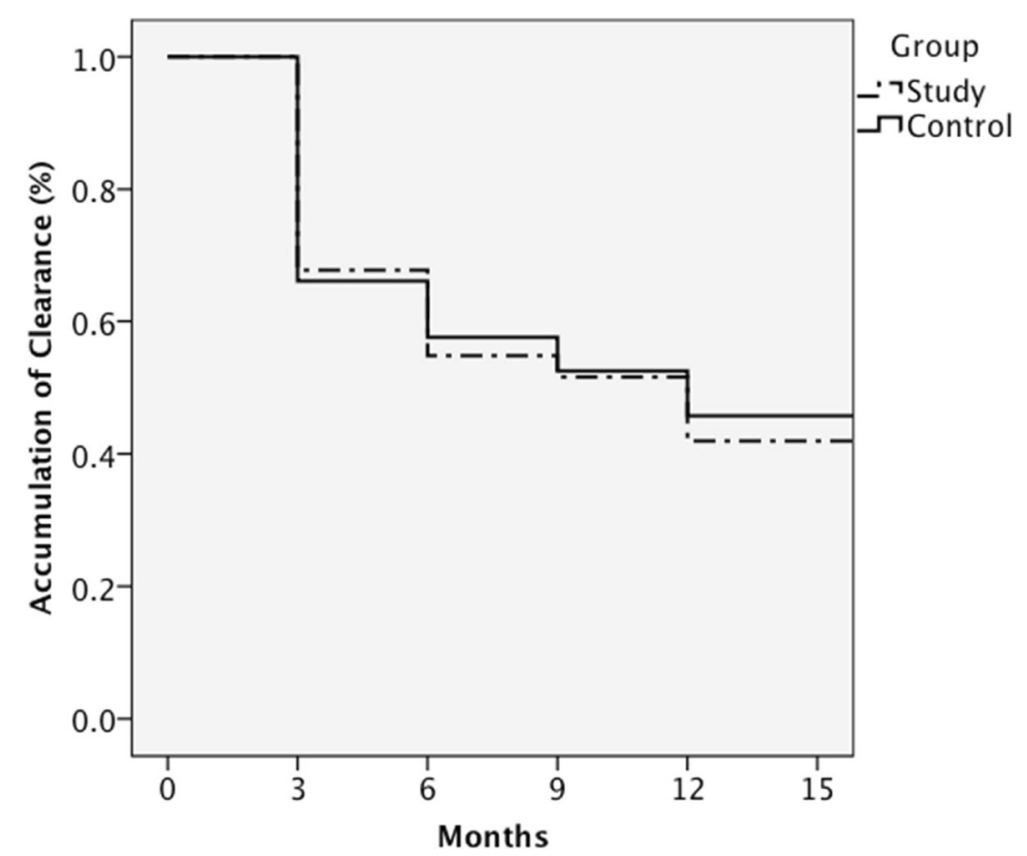

Fig. 2 Time to HR-HPV clearance; there was no significant difference between the two groups (log rank test, $p=0.741$ ) 
Table 2 Univariate and multivariate analyses of factors predicting HR-HPV clearance

\begin{tabular}{|c|c|c|c|c|c|}
\hline & HR-HPV clearance & & & Multivariate analysis & \\
\hline & Yes $(n=68)$ & No $(n=53)$ & $p$-value & $\mathrm{HR}(95 \% \mathrm{Cl})$ & $p$-value \\
\hline Age, years (mean $\pm S D$ ) & $44.72 \pm 10.50$ & $45.00 \pm 11.58$ & 0.890 & $0.494(0.092,2.654)$ & 0.623 \\
\hline Post-menopause & $21(30.9 \%)$ & $16(30.2 \%)$ & 0.986 & $1.007(0.451,2.251)$ & 0.848 \\
\hline Parity & $1.81 \pm 1.01$ & $2.28 \pm 1.25$ & 0.030 & $2.100(0.949,4.648)$ & 0.105 \\
\hline IUD & $8(11.8 \%)$ & $4(7.6 \%)$ & 0.752 & $1.469(0.405,5.326)$ & 0.734 \\
\hline Educational level (College or above) & $25(36.7 \%)$ & $14(26.4 \%)$ & 0.310 & $1.637(0.725,3.693)$ & 0.440 \\
\hline Employed & $44(64.7 \%)$ & $34(64.1 \%)$ & 0.846 & $1.107(0.513,2.386)$ & 0.449 \\
\hline History of $\mathrm{TH}$ & $4(5.9 \%)$ & $7(13.2 \%)$ & 0.104 & $0.260(0.065,1.032)$ & 0.147 \\
\hline Probiotics used & $36(52.9 \%)$ & $26(49.1 \%)$ & 0.716 & $1.168(0.569,397)$ & 0.672 \\
\hline Viral load (RLU/PC) (mean \pm SD) & $313.82 \pm 616.28$ & $561.88 \pm 868.58$ & 0.019 & $3.214(1.398,7.392)$ & 0.006 \\
\hline
\end{tabular}

Cl confidence interval, HR hazard ratio, HR-HPV high-risk human papillomavirus, IUD intra-uterine device, RLU/PC relative light unit/positive control, SD standard deviation, $T H$ total hysterectomy

and E7 oncogene expression in vitro [17]. The potent antiviral activity may in part explain the reduced risk of women acquiring sexually transmitted diseases, including HIV.

Two studies investigating the association between probiotics and HPV had been reported. In a recent trial, Palma et al. reported that HPV clearance was higher in the treatment of metronidazole plus 6 months vaginal Lactobacillus implementation than that with 3 months use, no control arm was included in that trial [18]. However, in a previous randomized pilot study, Verhoeven et al. failed to find any influence of probiotics on HPV clearance in a group of women with HPV-related LSILs of using oral Lactobacillus casei Shirota $\left(1 \times 10^{10} \mathrm{CFU} /\right.$ day) for 6 months [19]. Our report is the third study to evaluate the efficacy of probiotics on HR-HPV clearance. The population investigated in our study was somewhat different to that of Verhoeven et al., in that only $11 \%$ of our patients with HR-HPV infection had LSILs. We also failed to demonstrate an association between the use of probiotic strains and genital HR-HPV clearance. Although the findings of these studies have tended to be contradictory, there are still several issues that need to be clarified such as the dosage and duration of probiotic treatment, the route of administration, and the effect of probiotics on different types of HPV. Therefore, whether the role of probiotics in vaginal HPV infection is preventive, therapeutic, or both still requires further investigation.
Cervical smear screening is an effective method to detect cytological abnormalities. However, the quality of a smear is sometimes compromised by inflammatory cells and exudate, inadequate cellularity or failure to sample the transformation zone leading to an unsatisfactory result. A high unsatisfactory rate can increase the number of patient revisits, thereby increasing the overall cost of the screening program. Therefore how to lower the unsatisfactory rate has become an important issue before adopting cervical smear testing protocols into a large-scale screening program. The use of liquid-based cytology has been shown to be effective in reducing the unsatisfactory rate compared with conventional smears as it provides a cleaner smear though removing obscuring elements such as blood and inflammation [20]. The reduction has been shown to be greatest in younger women and to decrease with increasing age [21]. The reason may be due to lower cellularity with tissue atrophy in a non-estrogenic state following reduced ovarian function, which cannot be overcome by liquid base cytology [22]. It has been well established that probiotic strains can enable restoration and maintenance of normal vaginal flora and are thus helpful in the treatment and prevention of $\mathrm{BV}$ and vulvovaginal candidiasis $[8,9]$. This effect has also been shown in post-menopausal women $[23,24]$. A previous study showed that number of lactobacillus was increased following probiotic administration in postmenopausal women indicating low

Table 3 Distribution of cervical smear results before and after probiotic treatment between the two groups

\begin{tabular}{|c|c|c|c|c|c|}
\hline & \multicolumn{2}{|c|}{ Study Group $(N=62)$} & \multicolumn{2}{|c|}{ Control Group $(N=59)$} & \multirow[b]{2}{*}{$p$-value } \\
\hline & Pre-Treatment & Post-Treatment (6 months) & Pre-Treatment & Post-Treatment (6 months) & \\
\hline Normal & $13(20.9 \%)$ & $26(41.9 \%)$ & $18(30.5 \%)$ & $23(39.9 \%)$ & 0.672 \\
\hline Inflammation & $31(50 \%)$ & $30(41.9 \%)$ & $28(47.4 \%)$ & $26(44.1 \%)$ & 0.365 \\
\hline ASCUS and LSIL & $13(21.0 \%)$ & $4(6.5 \%)$ & $9(15.3 \%)$ & $6(10.2 \%)$ & 0.017 \\
\hline Unsatisfactory & $5(8.1 \%)$ & $2(3.2 \%)$ & $4(6.8 \%)$ & $4(6.8 \%)$ & 0.027 \\
\hline
\end{tabular}

ASCUS atypical squamous cells of undetermined significance, $L S I L$ low-grade squamous intraepithelial lesion 
estrogen levels wound not influence probiotic efficacy [25]. However whether the restoration of normal flora can increase cervical smear cellularity remains unknown. Therefore, the influence of probiotics on cervical smear quality is an interesting topic. Nevertheless, we only identified one related study in a literature search. Perisic et al. reported that the use of Lactobacillus rhamnosus GR-1 and Lactobacillus reuteri RC-14 could decrease the presence of unsatisfactory and/or borderline satisfactory cytological findings and thus provide a more reliable cytological diagnosis [26]. In our hospital, the annual Pap smear unsatisfactory rate is around $2.5 \%$, however in the present study the rate was as high as $7.44 \%$. There are two possible reasons for this finding. First, all cervical smears were obtained from conventional methods, and second, all of the participants had HR-HPV infections and BV was independently associated with HPV infection [5]. The high unsatisfactory rates in both arms just make our research feasible. We found a significant decrease in the unsatisfactory rate in the study arm, indicating the possible role of probiotics in improving cytological quality.

Another finding of this study was a significant decrease in the cervical smears showing mildly abnormalities (ASCUS/LSILs) in the women after taking probiotics. Although most of these mild abnormalities will regress without treatment, some may signal a precancerous condition or rarely cancer, especially in those positive for HR-HPV $[27,28]$. Verhoeven et al. evaluated women with LSIL-related HPV infections, and they also found a significantly higher cervical smear resolution rate in the probiotic group [19]. Since a LSIL is not a surrogate predictor of cervical cancer and as there was no impact on HR-HPV clearance, there is currently no conclusive clinical evidence of the effect of probiotics on cancer prevention, although in-vitro and animal studies have provided such evidence [29].

Several studies have shown that viral load measurements of HR-HPV types in cervical specimens can be a suitable indicator of viral clearance or persistence [30,31]. In the present study, we also found that a low viral load was the only independent factor predicting HPV clearance. Although estimation of HR-HPV viral load by HC-II has been shown to correlate well with viral load estimated by real-time PCR [32], multiple infections, cross-reactivity of nononcogenic HPV types, and variability in the cellularity of cervical samples may have biased the results. Therefore, the HC-II assay has only been validated as a semiquantitative test for HR-HPV viral load measurement.

There are several limitations to this study. First, substantial risk factors for cervical cancer such as smoking, number of sexual partners, and the use of oral contraceptives were not included for analysis, and this may have led to some form of bias. Second, status of BV and vaginal $\mathrm{pH}$, which are thought to be important variables determining HPV clearance, were not evaluated in our study. Third, the sample size was small after adjustment because of slow recruitment and also the follow-up period was short. Fouth, HPV typing and quantitative real-time PCR assays were not applied since viral clearance may differ among various HPV types and the accuracy of quantitative PCR in viral load measurement is greater than that of $\mathrm{HC}$-II.

\section{Conclusion}

We demonstrated that the application of the probiotic strains Lactobacillus rhamnosus GR-1 and Lactobacillus reuteri $\mathrm{RC}-14$ provided no influence on genital HR-HPV clearance but may have decreased the mildly abnormal and unsatisfactory rates of cervical smears. A larger well-designed randomized study is warranted to confirm these results.

\section{Abbreviations}

ASCUS: Atypical squamous cell of undetermined significance; BV: Bacterial vaginosis; HC: Hybrid capture; HR-HPV: High risk human papilloma virus; LSIL: Low grade squamous intraepithelial lesion; PCR: Polymerase chain reaction

\section{Acknowledgments}

The authors thank all of the volunteers for participating in this study, and also Tri-factor Biotech Inc., Taiwan for providing probiotic and placebo capsules for this clinical trial. Tri-Factor Biotech Inc., Taiwan were not involved in any aspect of the clinical trial including the design, conduct, and analysis of the results.

\section{Authors' contributions}

YCO and HL were involved in the study conception and design. CHW, CCT, and CWT conducted the acquisition of data. HCF performed the data statistical analysis. YCO and HCF prepare the manuscript. HL revised the manuscript for quality and consistency of the data. All authors have read and approved the final version of the manuscript.

\section{Funding}

This study including its data collection, analysis and interpretation, as well as manuscript preparation was financially supported by grant CMRPG890891, from Chang Gung Memorial Hospital, Taiwan.

Availability of data and materials

The data are available upon reasonable request from the corresponding author.

Ethics approval and consent to participate

This study was approved by the Institutional Review Board of Chang Gung Memorial Hospital, under Protocol number 99-0948A3. All participants gave their informed written consent to take part in the study.

Consent for publication

Not applicable.

\section{Competing interests}

The authors declare that they have no competing interests.

\section{Author details}

${ }^{1}$ Department of Obstetrics and Gynecology, Chia-Yi Chang Gung Memorial Hospital, Chia-Yi, Taiwan. ${ }^{2}$ Department of Obstetrics and Gynecology, Kaohsiung Chang Gung Memorial Hospital and Chang Gung University College of Medicine, 123, Ta Pei Road, Niao Sung District, Kaohsiung City 83301, Taiwan, Republic of China. 
Received: 1 January 2019 Accepted: 3 July 2019

Published online: 24 July 2019

\section{References}

1. Hawes SE, Hillier SL, Benedetti J, Stevens CE, Koutsky LA, Wolner-Hanssen P, Holmes KK. Hydrogen peroxide-producing lactobacilli and acquisition of vaginal infections. J Infect Dis. 1996;174(5):1058-63.

2. Gupta K, Stapleton AE, Hooton TM, Roberts PL, Fennell CL, Stamm WE. Inverse association of $\mathrm{H}_{2} \mathrm{O} 2$-producing lactobacilli and vaginal Escherichia coli colonization in women with recurrent urinary tract infections. J Infect Dis. 1998;178(2):446-50.

3. da Silva CS, Adad SJ, Hazarabedian de Souza MA, Macedo Barcelos AC, Sarreta Terra AP, Murta EF. Increased frequency of bacterial vaginosis and chlamydia trachomatis in pregnant women with human papillomavirus infection. Gynecol Obstet Investig. 2004;58(4):189-93.

4. Mao C, Hughes JP, Kiviat N, Kuypers J, Lee SK, Adam DE, Koutsky LA. Clinical findings among young women with genital human papillomavirus infection. Am J Obstet Gynecol. 2003;188(3):677-84.

5. Gillet E, Meys JF, Verstraelen H, Bosire C, De Sutter P, Temmerman M, Broeck DV. Bacterial vaginosis is associated with uterine cervical human papillomavirus infection: a meta-analysis. BMC Infect Dis. 2011;11:10.

6. Castellsague $X$. Natural history and epidemiology of HPV infection and cervical cancer. Gynecol Oncol. 2008;110(3 Suppl 2):S4-7.

7. Huh WK. Human papillomavirus infection: a concise review of natural history. Obstet Gynecol. 2009;114(1):139-43.

8. Reid G, Bruce AW, Fraser N, Heinemann C, Owen J, Henning B. Oral probiotics can resolve urogenital infections. FEMS Immunol Med Microbiol. 2001;30(1):49-52.

9. Cianci A, Giordano R, Delia A, Grasso E, Amodeo A, De Leo V, Caccamo F. Efficacy of Lactobacillus Rhamnosus GR-1 and of Lactobacillus Reuteri RC-14 in the treatment and prevention of vaginoses and bacterial vaginitis relapses. Minerva Ginecol. 2008;60(5):369-76.

10. Chew SY, Cheah YK, Seow HF, Sandai D, Than LT. Probiotic Lactobacillus rhamnosus GR-1 and Lactobacillus reuteri RC-14 exhibit strong antifungal effects against vulvovaginal candidiasis-causing Candida glabrata isolates. J Appl Microbiol. 2015;118(5):1180-90.

11. Ho M, Chang YY, Chang WC, Lin HC, Wang MH, Lin WC, Chiu TH. Oral Lactobacillus rhamnosus GR-1 and Lactobacillus reuteri RC-14 to reduce group B Streptococcus colonization in pregnant women: a randomized controlled trial. Taiwan J Obstet Gynecol. 2016;55(4):515-8.

12. Reid G. Probiotic agents to protect the urogenital tract against infection Am J Clin Nutr. 2001;73(2 Suppl):437S-43S.

13. Anukam KC, Osazuwa EO, Osadolor HB, Bruce AW, Reid G. Yogurt containing probiotic Lactobacillus rhamnosus GR-1 and L. reuteri RC-14 helps resolve moderate diarrhea and increases CD4 count in HIV/AIDS patients. J Clin Gastroenterol. 2008;42(3):239-43.

14. Anukam KC, Hayes K, Summers K, Reid G. Probiotic Lactobacillus rhamnosus GR-1 and Lactobacillus reuteri RC-14 may help downregulate TNF-alpha, IL$6, I L-8, I L-10$ and IL-12 (p70) in the neurogenic bladder of spinal cord injured patient with urinary tract infections: a two-case study. Adv Urol. 2009;2009:680363.

15. Van Baarlen P, Troost F, van der Meer C, Hooiveld G, Boekschoten M, Brummer RJ, Kleerebezem M. Human mucosal in vivo transcriptome responses to three lactobacilli indicate how probiotics may modulate human cellular pathways. Proc Natl Acad Sci U S A. 2011;108:4562-9.

16. Cadieux P, Burton J, Gardiner G, Braunstein I, Bruce AW, Kang CY, Reid G. Lactobacillus strains and vaginal ecology. JAMA. 2002;287(15):1940-1.

17. Cha MK, Lee DK, An HM, Lee SW, Shin SH, Kwon JH, Kim KJ, Ha NJ. Antiviral activity of Bifidobacterium adolescentis SPM1005-a on human papillomavirus type 16. BMC Med. 2012;10:72.

18. Palma E, Recine N, Domenici L, Giorgini M, Pierangeli A, Panici PB. Longterm Lactobacillus rhamnosus BMX 54 application to restore a balanced vaginal ecosystem: a promising solution against HPV-infection. BMC Infect Dis. 2018;18(1):13

19. Verhoeven V, Renard N, Makar A, Van Royen P, Bogers JP, Lardon F, et al. Probiotics enhance the clearance of human papillomavirus-related cervical lesions: a prospective controlled pilot study. Eur J Cancer Prev. 2013;22(1): 46-51.

20. Akamatsu S, Kodama S, Himeji Y, Ikuta N, Shimagaki N. A comparison of liquid-based cytology with conventional cytology in cervical cancer screening. Acta Cytol. 2012;56(4):370-4.
21. Castle PE, Bulten J, Confortini M, Klinkhamer P, Pellegrini A, Siebers AG, et al. Age-specific patterns of unsatisfactory results for conventional pap smears and liquid-based cytology: data from two randomised clinical trials. BJOG. 2010;117(9):1067-73.

22. Siebers AG, Klinkhamer PJ, Vedder JE, Arbyn M, Bulten J. Causes and relevance of unsatisfactory and satisfactory but limited smears of liquidbased compared with conventional cervical cytology. Arch Pathol Lab Med. 2012;136(1):76-83.

23. Kwak YK, Daroczy K, Colque P, Kühn I, Möllby R, Kopp Kallner H. Persistence of lactobacilli in postmenopausal women - a double-blind, randomized, pilot study. Gynecol Obstet Investig. 2017;82(2):144-50.

24. Kim JM, Park YJ. Probiotics in the pevention and treatment of postmenopausal vaginal infections: review article. J Menopausal Med. 2017; 23(3):139-45.

25. Bisanz JE, Seney S, McMillan A, Vongsa R, Koenig D, Wong L, et al. A systems biology approach investigating the effect of probiotics on the vaginal microbiome and host responses in a double blind, placebo-controlled clinical trial of post-menopausal women. PLoS One. 2014;9(8):e104511.

26. Perisic Z, Perisic N, Golocorbin Kon S, Vesovic D, Jovanovic AM, Mikov M. The influence of probiotics on the cervical malignancy diagnostics quality. Vojnosanit Pregl. 2011;68(11):956-60.

27. Stoler MH, Wright TC Jr, Sharma A, Apple R, Gutekunst K, Wright TL; ATHENA (Addressing THE Need for Advanced HPV Diagnostics) HPV Study Group. High-risk human papillomavirus testing in women with ASC-US cytology: results from THE ATHENA HPV study. Am J Clin Pathol 2011;135(3): 468-475.

28. Arbyn M, Xu L, Verdoodt F, Cuzick J, Szarewski A, Belinson JL, et al. Genotyping for human papillomavirus types 16 and 18 in women with minor cervical lesions: a systematic review and meta-analysis. Ann Intern Med. 2017;166(2):118-27.

29. Nanno M, Kato I, Kobayashi T, Shida K. Biological effects of probiotics: what impact does Lactobacillus casei shirota have on us? Int J Immunopathol Pharmacol. 2011;24(1 Suppl):45S-50S

30. van der Weele $P$, van Logchem E, Wolffs $P$, van den Broek I, Feltkamp M, de Melker $\mathrm{H}$, et al. Correlation between viral load, multiplicity of infection, and persistence of HPV16 and HPV18 infection in a Dutch cohort of young women. J Clin Virol. 2016;83:6-11.

31. Trevisan A, Schlecht NF, Ramanakumar AV, Villa LL, Franco EL, Ludwig-McGill Study Group. Human papillomavirus type 16 viral load measurement as a predictor of infection clearance. J Gen Virol. 2013;94(Pt 8):1850-7.

32. Prétet JL, Dalstein V, Monnier-Benoit S, Delpeut S, Mougin C. High risk HPV load estimated by hybrid capture II correlates with HPV16 load measured by real-time PCR in cervical smears of HPV16-infected women. J Clin Virol. 2004:31(2):140-7.

\section{Publisher's Note}

Springer Nature remains neutral with regard to jurisdictional claims in published maps and institutional affiliations.

\section{Ready to submit your research? Choose BMC and benefit from:}

- fast, convenient online submission

- thorough peer review by experienced researchers in your field

- rapid publication on acceptance

- support for research data, including large and complex data types

- gold Open Access which fosters wider collaboration and increased citations

- maximum visibility for your research: over $100 \mathrm{M}$ website views per year

At $\mathrm{BMC}$, research is always in progress.

Learn more biomedcentral.com/submissions 